\title{
KIF15 Expression in Tumor-associated Monocytes Is a Prognostic Biomarker in Hepatocellular Carcinoma
}

\author{
AKIHIRO KITAGAWA ${ }^{1,2}$, TAKAAKI MASUDA ${ }^{1}$, JUNICHI TAKAHASHI ${ }^{1}$, TARO TOBO $^{3}$, MIWA NODA $^{1}$, \\ YOSUKE KURODA $^{1}$, QINGJIANG HU ${ }^{1}$, YUTA KOUYAMA ${ }^{1}$, YUTA KOBAYASHI ${ }^{1,2}$, SHOTARO KURAMITSU ${ }^{1}$, \\ KUNIAKI SATO $^{1}$, ATSUSHI FUJII ${ }^{1}$, YUKIHIRO YOSHIKAWA ${ }^{1,2}$, HIROAKI WAKIYAMA ${ }^{1}$, DAI SHIMIZU ${ }^{1}$, \\ YUSUKE TSURUDA ${ }^{1}$, HIDETOSHI EGUCHI $^{2}$, YUICHIRO DOKI ${ }^{2}$, MASAKI MORI $^{4}$ and KOSHI MIMORI ${ }^{1}$ \\ Departments of ${ }^{1}$ Surgery and ${ }^{3}$ Clinical Laboratory Medicine, Kyushu University, Beppu Hospital, Oita, Japan; \\ ${ }^{2}$ Department of Gastroenterological Surgery, Graduate School of Medicine, Osaka University, Osaka, Japan; \\ ${ }^{4}$ Department of Surgery and Science, Graduate School of Medical Science, Kyushu University, Fukuoka, Japan
}

\begin{abstract}
Background/Aim: Kinesin family member 15 (KIF15) participates in the transport of macromolecules in essential cellular processes. In this study we evaluated the clinical relevance of KIF15 expression in hepatocellular carcinoma (HCC). Materials and Methods: Association between KIF15 expression and clinical outcomes in HCC patients was analyzed using three independent cohorts. Localization of KIF15 expression was assessed by immunohistochemical analysis. Co-culture experiments were performed using healthy donor peripheral blood mononuclear cells (PBMC) and HCC cell lines. Results: Immunohistochemical analysis showed that KIF15 was mainly expressed in inflammatory monocytes around cancer cells. Multivariate analysis indicated high KIF15 expression was an independent poor prognostic factor for survival. HCC cells with high expression of minichromosome maintenance protein 2 (MCM2) were located close to KIF15expressing inflammatory monocytes. The proliferation ability of HCC cells was increased by co-culture with PBMC. Conclusion: High KIF15 expression in inflammatory monocytes in tumor tissues may serve as a prognostic marker for poor outcome in HCC.
\end{abstract}

Hepatocellular carcinoma (HCC) is the fifth most common cancer and the third leading cause of cancer-related deaths worldwide $(1,2)$. HCC is essentially an incurable malignancy

This article is freely accessible online.

Correspondence to: Koshi Mimori, MD, Ph.D., Department of Surgery, Kyushu University, Beppu Hospital, 4546 Tsurumihara, Beppu, Oita, 874-0838, Japan. Tel: +81 977271650, Fax: +81 977271651, e-mail: kmimori@beppu.kyushu-u.ac.jp

Key Words: KIF15, hepatocellular carcinoma, intracellular transporter, inflammatory monocyte, biomarker. due to the limited availability of radical therapeutic approaches. In most cases, the only potentially curative treatment remains surgical resection and liver transplantation (3). It should be important to clarify the molecular mechanisms involved in HCC progression and identify novel prognostic molecular biomarkers of HCC to achieve improvements in survival.

Intracellular transport is fundamental for cellular function, survival and morphogenesis. After synthesis, cells transport and sort proteins and lipids to various destinations at appropriate velocities in organelles and protein complexes (4). Among the molecular motors that are involved intracellular transport, 3 large superfamilies have been identified, including kinesins, dyneins and myosins $(5,6)$. In the kinesin superfamily (KIF) of proteins, one of those motors for anterograde transport of mitochondria, comprise a group of proteins that share a highly conserved motor domain and facilitate the transport of mRNA, protein complexes and organelles in an ATP-and microtubule-dependent manner (4, 7,8). Previous studies revealed that many KIFs are involved in carcinogenesis, invasion and metastasis of multiple human cancers, including HCC (9-12).

KIF15, which is the second tetrameric spindle motor (in addition to kinesin-5, Eg5), modulates spindle microtubule architecture with Tpx2 and drives centrosome separation during bipolar spindle assembly (13-15). KIF15 also plays an important role in tumor progression, as previously described $(9,16,17)$. For instance, KIF15 is considered a key regulator that promotes $G_{1} / S$ phase transition via regulating the MEKERK signaling pathway. It represents a new therapeutic target for pancreatic cancer, although a detailed mechanism of KIF15 function is lacking (9). However, the clinical and biological significance of KIF15 expression in HCC has not been fully elucidated. In this study, we aimed to determine the clinical significance of KIF15 expression in HCC and investigated its biological role in HCC progression. 


\section{Materials and Methods}

Public datasets. The Cancer Genome Atlas database (TCGA) via Broad Institute's Firehose (http://gdac.broadinstitute.org/) and GSE14520 dataset via the GEO database (http:// www.ncbi.nlm.nih.gov/) were analyzed in this study. There were mRNA expression data from 371 tumor tissues and 50 non-cancerous tissues in TCGA dataset and 247 tumor tissues and 239 noncancerous tissues in the GSE14520 dataset. TCGA and GSE14520 sequencing data were normalized with quantile normalization (18).

Gene set enrichment analysis. The associations between KIF15 expression and previously defined gene sets were analyzed by gene set enrichment analysis (GSEA) using HCC expression profiles from the appropriate TCGA dataset. The biologically defined gene sets were obtained from the Molecular Signatures Database v5.2 (http://software.broadinstitute.org/gsea/msigdb/index.jsp).

Patients and sample collection from our patient cohort. Overall, 59 patients with HCC who underwent liver resection at the Kyushu University Beppu Hospital and its affiliated hospitals between 2000 and 2004 were enrolled in this study, as previously described (1921). Tissues from resected tumors were immediately stored in RNAlater (Ambion, Austin, TX, USA), frozen in liquid nitrogen and kept at $-80^{\circ} \mathrm{C}$ until RNA extraction. Corresponding noncancerous liver tissues were also collected (available in 49 of 59 cases). A 5-year follow-up was conducted after surgery, and the average follow-up period for the 59 patients was 60.0 months (range $=3.0-60.0$ months).

Cell lines and cell culture. Established human hepatocellular carcinoma cell lines (HepG2 and HuH7), a monocyte cell line (THP1) and a fibroblast cell line (KMST-6) were purchased from the American Type Culture Collection. These cell lines were cultured in D-MEM (HepG2 and HuH7) or RPMI1640 (THP1 and KMST-6) (Wako, Osaka, Japan) supplemented with $10 \%$ fetal bovine serum (FBS) at $37^{\circ} \mathrm{C}$ in a humidified atmosphere containing $5 \% \mathrm{CO}_{2}$.

RNA extraction. Total RNA was extracted from frozen tissue samples, cell lines and healthy donor peripheral blood mononuclear cells (PBMC) using Isogen (Nippon Gene, Tokyo, Japan), as previously described (22).

Reverse transcription-quantitative $P C R \quad(R T-q P C R)$. RT was performed using M-MLV reverse transcriptase (Invitrogen, Carlsbad, CA, USA), as previously described $(23,24)$, and the quantitative polymerase chain reaction (qPCR) was performed using a LightCycler 480 SYBR Green I Master Mix (Roche, Basel, Switzerland) as previously described $(23,24)$. The following primers were used: KIF15: 5'-AATTTGCGGCCATCTTGAGC-3' (sense) and 5'-CTGACCATTTGTCACGCTGC-3' (antisense); GAPDH: 5'AGCCACATCGCTCAGACAC-3' (sense) and 5'-GCC CAATACG ACCAAATCC-3' (antisense). The expression level of KIF15 mRNA was normalized to that of GAPDH.

Western blot analysis. Western blot analysis was performed as previously described $(22,25)$. The following antigen-specific antibodies were used: primary goat polyclonal antibody against MCM2 (sc-9839; Santa Cruz Biotechnology, Santa Cruz, CA, USA) at a dilution of 1:500; and, primary mouse polyclonal antibody against $\beta$-actin (Santa Cruz Biotechnology, Santa Cruz, CA, USA) at a dilution of 1:1,000. Expression of MCM2 proteins was normalized to the expression of $\beta$-actin.

Isolation of healthy donor PBMCs. PBMCs in 30 to $40 \mathrm{ml}$ of blood were obtained from a healthy donor. The cells were collected in EDTA tubes and they were isolated using Ficoll-Paque PLUS (GE Healthcare, Japan) density gradient centrifugation.

PBMC culture and co-culture of HCC cell lines with PBMC. The isolated PBMCs were re-suspended in culture medium containing DMEM (Wako, Osaka, Japan) supplemented with $10 \%$ fetal bovine serum (FBS) and $100 \mathrm{U} / \mathrm{ml}$ Pen/Strep, and PBMCs were enumerated by cell count. The above culture medium was chosen after experiments that were performed with both HCC cell lines (HepG2 and $\mathrm{HuH7}$ ) and PBMCs in order to find optimal co-culture conditions. Therefore, the control HCC cell lines were maintained in monocultures using the same medium. The viability of PBMCs after 7-14 days in culture always exceeded $30-50 \%$.

We co-cultured HCC cell lines with PBMCs plated on $10-\mathrm{cm}$ culture dishes (Thermo Fisher Scientific, USA). Cells were cocultured on culture inserts (FALCON, USA) for 7-10 days such that $6 \times 10^{3} \mathrm{HepG} 2$ were mixed with $1 \times 10^{5} \mathrm{PBMC}$ or $6 \times 10^{3} \mathrm{HuH} 7$ were mixed with $1 \times 10^{5} \mathrm{PBMC}$. Total RNA from co-cultured HCC cell lines (HepG2 and $\mathrm{HuH} 7)$ and PBMC were extracted using ISOGEN (Nippon Gene, Tokyo, Japan). For co-culture experiments, HepG2 and $\mathrm{HuH} 7$ cells were plated at a density of 3000 cells/well in triplicate onto 6-well plates and incubated at $37^{\circ} \mathrm{C}$ under $5 \% \mathrm{CO}_{2}$ with antibiotic selection.

Colony formation assay. After 10 days, the colonies were stained using a Differential Quick Stain Kit (Sysmex) according to the manufacturer's instructions. Visible colonies were photographed using a FUSION SOLO S. Colony counts were determined using the ImageJ software (version 1.80, NIH, Bethesda, MD, USA).

MTT assay. MTT assays were conducted using a Cell Proliferation Kit I (Roche Applied Science) to evaluate cell proliferation following the manufacturer's instructions, as previously described (22).

Immunohistochemical analysis (IHC). Immunohistochemical analysis of KIF15 expression was performed with formalin-fixed paraffinembedded surgical sections (FFPE) obtained from patients with HCC, or a HCC cell line (HepG2) or healthy donor PBMC, as previously described $(21,26)$. Primary rabbit polyclonal antibody against KIF15 (ab90735; Abcam) was used at a dilution of 1:200. Histological analysis was performed independently by an experienced research pathologist at the Kyushu University.

Immunofluorescence analysis (IF). Antibodies against KIF15 (antirabbit IgG 555, Cell Signaling) and MCM2 (anti-mouse IgG 488, Cell Signaling) were used to stain KIF15 protein and MCM2 protein, respectively in FFPE, at a dilution of 1:1,000 and 1:250, as described above and fluorescent images were observed using a fluorescence microscope (BZ-X700, Keyence, Osaka, Japan).

We counted the number of inflammatory monocytes and HCC cells stained clearly by antibody against KIF15 and MCM2 divided into several segments in a field of view at $\times 200$ and examined the correlation between them. 
A

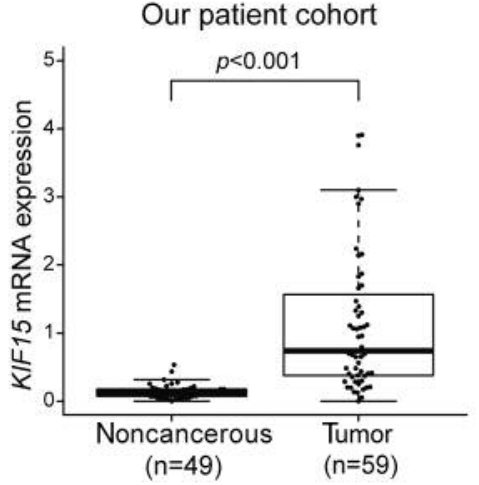

B
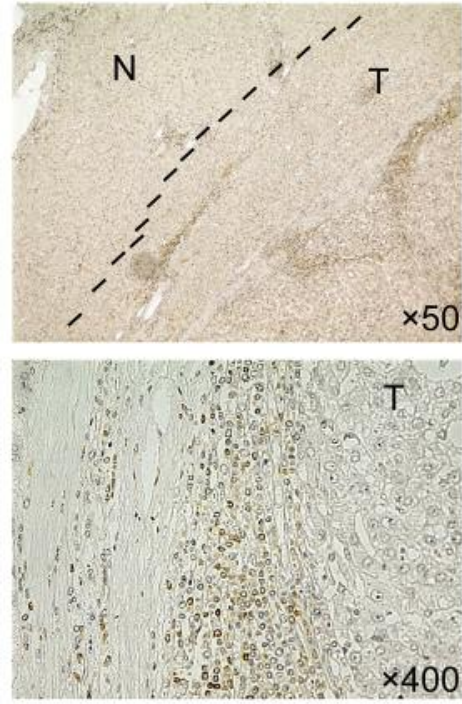

D

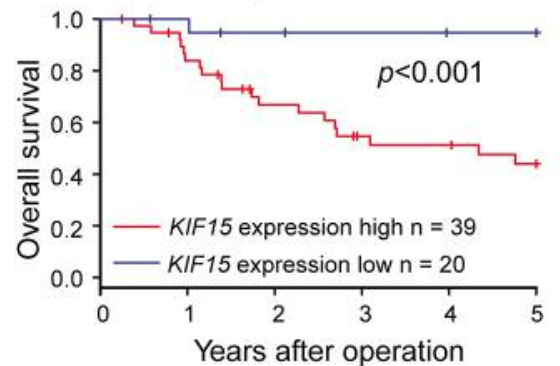

TCGA dataset
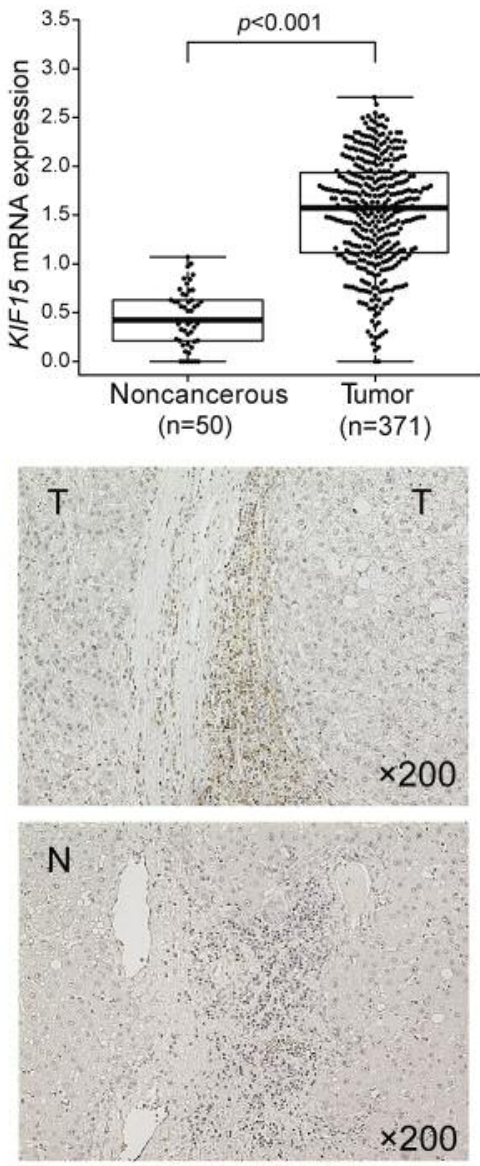

TCGA dataset

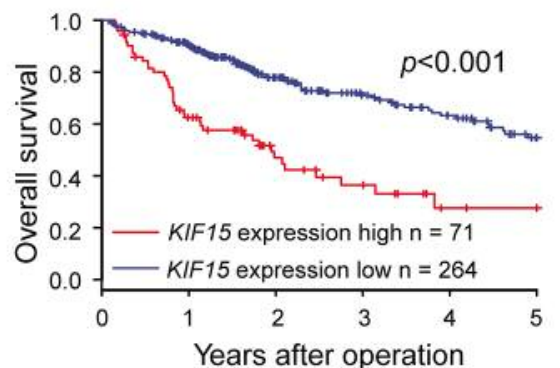

GSE14520 dataset

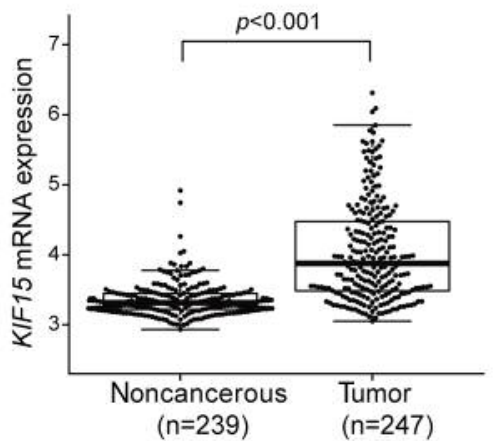

C

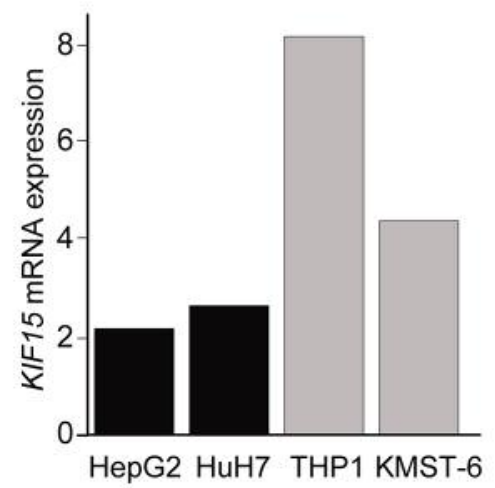

E

HALLMARK_MYC_TARGETS_V1

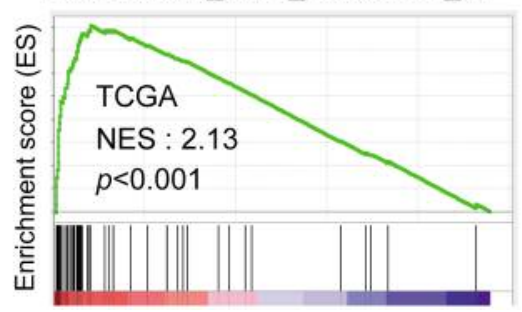

HALLMARK E2F_TARGETS

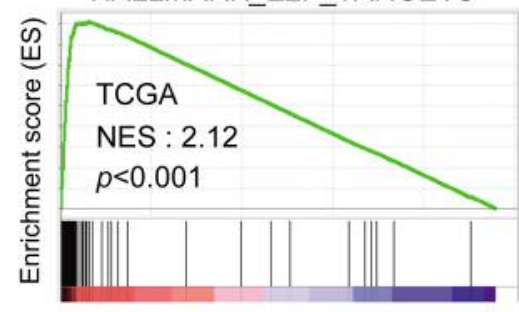

Figure 1. Clinical significance of KIF15 expression in HCC tissues. (A) KIF15 mRNA expression in tumor tissues and non-cancerous tissues in our HCC patient cohort, TCGA dataset and GSE14520 dataset. (B) Immunohistochemical detection of KIF15 expression in a representative sample of HCC. Original magnifications: $\times 50, \times 200$ and $\times 400 .(C)$ KIF15 mRNA expression in HCC cell lines (HepG2 and HuH7) and monocyte and fibroblast cell lines (THP1 and KMST-6, respectively). (D) Kaplan-Meier survival curve of 59 HCC patients from our patient cohort, 335 HCC patients from a TCGA dataset, and 242 HCC patients from the GSE14520 dataset based on KIF15 mRNA expression. (E) GSEA of HCC cases from a TCGA dataset. 
Table I. Univariate and multivariate analyses of clinicopathologic factors for overall survival in our HCC cases $(N=59)$.

\begin{tabular}{|c|c|c|c|c|c|c|}
\hline \multirow[t]{2}{*}{ Variable } & \multicolumn{3}{|c|}{ Univariate analysis } & \multicolumn{3}{|c|}{ Multivariate analysis } \\
\hline & HR & $95 \% \mathrm{CI}$ & $p$-Value & $\mathrm{HR}$ & $95 \% \mathrm{CI}$ & $p$-Value \\
\hline Age $\geq 65$ (years) & 0.6 & $0.24-1.60$ & 0.294 & & & \\
\hline Gender (male/female) & 1.93 & $0.7-6.76$ & 0.209 & & & \\
\hline Hepatitis B (present/absent) & 1.3 & $0.46-3.26$ & 0.594 & & & \\
\hline Hepatitis C (present/absent) & 1.21 & $0.49-3.23$ & 0.673 & & & \\
\hline Child-Pugh classification (A/B and C) & 1.13 & $0.26-3.37$ & 0.845 & & & \\
\hline $\mathrm{AFP} \geq \operatorname{median}(\mathrm{ng} / \mathrm{ml})$ & 4.18 & $1.59-13.03$ & $0.003^{*}$ & 2.33 & $0.69-8.43$ & 0.17 \\
\hline Maximum tumor size $\geq 3(\mathrm{~cm})$ & 5.44 & $2.07-16.93$ & $<0.001^{*}$ & 3.57 & $1.14-13.57$ & $0.02 *$ \\
\hline Number of tumors (single/multiple) & 1.49 & $0.55-3.64$ & 0.405 & & & \\
\hline Portal vein invasion (present/absent) & 1.55 & $0.58-4.12$ & 0.537 & & & \\
\hline Hepatic vein invasion (present/absent) & 1.48 & $0.55-3.99$ & 0.495 & & & \\
\hline Biliary invasion (present/absent) & 2.78 & $0.56-50.17$ & 0.225 & & & \\
\hline Histological differentiationa (well, moderately/poorly) & 4.11 & $1.43-10.60$ & $0.011^{*}$ & 0.87 & $0.27-2.88$ & 0.82 \\
\hline Expression (low/high) & 13.39 & $2.76-240.91$ & $<0.001^{*}$ & 8.83 & $1.66-163.31$ & $0.006^{*}$ \\
\hline
\end{tabular}

aWHO grades 1 and 2 or Edmondson-Steiner grade 1 and 2 were classified as well-differentiated or moderately differentiated. WHO grades 3 and 4 or Edmondson-Steiner grade 3 and 4 were classified as poorly differentiated. HR: Hazard ratio, CI: confidence interval, HBV: hepatitis B virus, HCV: hepatitis C virus, AFP: $\alpha$-fetoprotein, HCC: hepatocellular carcinoma, WHO: World Health Organization. *Indicates statistical significance.

Statistical analysis. For clinical analysis, cases were divided into 2 groups using the minimum $p$-value approach based on the KIF 15 expression level, which is a comprehensive method to identify the optimal risk separation cut-off point in continuous gene expression measurements for survival analysis in multiple datasets (27). For continuous variables, data were expressed as means \pm standard deviation, and statistical analysis was performed using Welch's $t$-tests. The degree of linearity was estimated by Spearman's rank correlation coefficient. Categorical variables were compared using chi square tests or Fisher's exact tests. Overall survival (OS) was estimated using the Kaplan-Meier method, and the survival curves were compared using the log-rank test. Univariate and multivariate analyses were performed using the Cox proportional hazards model to identify independent variables predictive of OS. $p<0.05$ was considered statistically significant. Data analysis of clinicopathological factors was performed using the JMP 13 software (SAS Institute, Cary, NC, USA), and other analyses were performed using the R version 3.1.1 (Vienna, Austria; http://www.R-project.org/).

\section{Results}

KIF15 expression was up-regulated in human HCC tissues. First, we analyzed KIF 15 mRNA expression in HCC tissues and non-cancerous liver tissues using 2 public data sources (TCGA and GSE14520 datasets). Moreover, we measured KIF15 mRNA expression by RT-qPCR using 59 tumor tissues and 49 paired non-cancerous tissues from HCC patients in our hospital. We found that KIF 15 mRNA expression in HCC tissues was significantly higher compared to non-cancerous tissues in our cohort and in two public databases $(p<0.001$, Figure 1A). Next, immunohistochemical analysis showed that KIF15 was mainly stained in the inflammatory monocytes infiltrating closely to cancer cells compared with noncancerous tissues (Figure 1B). This is consistent with the fact that KIF15 mRNA expression level is lower in HCC cell lines (HepG2 and HuH7) compared to its expression in a monocyte cell line (THP1) and a fibroblast cell line (KMST-6) (Figure 1C). These results suggest that KIF15 is expressed mainly in tumor-associated monocytes in HCC.

Prognostic significance of KIF15 expression in HCC tissues. We performed prognostic analyses of KIF15 expression in 3 datasets (our patient cohort, a TCGA dataset, and the GSE15420 dataset). With each dataset, we divided the total cases into high- and low- KIF15 expression groups using the minimum $p$-value approach based on the KIF15 expression level, as described in the Materials and Methods section. Overall survival of the high KIF15 expression group was significantly poorer than that in the low-expression group in our patient cohort (log-rank test, $\mathrm{n}=59, p<0.01$ ), TCGA dataset (log-rank test, $\mathrm{n}=335, p<0.001)$ and the GSE14520 dataset (log-rank test, $\mathrm{n}=242, p<0.001$ ) (Figure 1D).

Then, we performed a multivariate analysis with all 4 variables affecting OS in univariate analyses in our patient cohort (KIF15 mRNA expression in HCC, AFP, maximum tumor size and histological differentiation). Of note, high KIF 15 expression was an independent prognostic factor for poor outcome $(p<0.01, \mathrm{HR}=8.83,95 \% \mathrm{CI}=1.66-163.31)$ (Table I).

Clinicopathological significance of KIF15 expression in HCC tissues. The relationship between clinicopathological factors 
Table II. KIF15 expression and clinicopathological factors in our HCC cases $(N=59)$.

\begin{tabular}{|c|c|c|c|}
\hline \multirow[t]{2}{*}{ Factors } & \multicolumn{2}{|c|}{ KIF15 mRNA expression } & \multirow[t]{2}{*}{$p$-Value } \\
\hline & Low $(n=20)$ & High $(n=39)$ & \\
\hline Age, years $($ mean \pm SD) & $67.9 \pm 6.6$ & $64.9 \pm 10.0$ & 0.239 \\
\hline Gender & & & 0.131 \\
\hline Male & 17 & 25 & \\
\hline Female & 3 & 14 & \\
\hline Etiology of HBV infection & & & 0.765 \\
\hline Present & 5 & 12 & \\
\hline Absent & 15 & 27 & \\
\hline Etiology of HCV infection & & & 0.780 \\
\hline Present & 11 & 24 & \\
\hline Absent & 9 & 15 & \\
\hline Child-Pugh classification & & & 1.000 \\
\hline A & 25 & 27 & \\
\hline $\mathrm{B}$ and $\mathrm{C}$ & 4 & 3 & \\
\hline $\operatorname{AFP}(\mu \mathrm{g} / 1)$ & & & 0.051 \\
\hline$<$ median & 14 & 15 & \\
\hline$\geq$ median & 6 & 23 & \\
\hline NA & 1 & & \\
\hline \multicolumn{4}{|l|}{ Maximum tumor size $(\mathrm{cm})$} \\
\hline$<3$ & 17 & 28 & $0.012 *$ \\
\hline$\geq 3$ & 3 & 11 & \\
\hline Number of tumors & & & 0.765 \\
\hline Sigle & 15 & 27 & \\
\hline Multiple & 5 & 12 & \\
\hline Portal vein invasion & & & 0.059 \\
\hline Present & 3 & 16 & \\
\hline Absent & 13 & 16 & \\
\hline NA & 4 & 7 & \\
\hline Hepatic vein invasion & & & 1.000 \\
\hline Present & 8 & 16 & \\
\hline Absent & 8 & 16 & \\
\hline NA & 4 & 7 & \\
\hline Bile duct invasion & & & 0.366 \\
\hline Present & 13 & 29 & \\
\hline Absent & 3 & 3 & \\
\hline NA & 4 & 7 & \\
\hline Histological differentiationa & & & $0.040 *$ \\
\hline Well or moderately & 18 & 27 & \\
\hline Poorly & 0 & 8 & \\
\hline NA & 2 & 4 & \\
\hline
\end{tabular}

aWHO grades 1 and 2 or Edmondson-Steiner grade 1 and 2 were classified as well-differentiated or moderately differentiated. WHO grades 3 and 4 or Edmondson-Steiner grade 3 and 4 were classified as poorly differentiated. SD: Standard deviation, HBV: hepatitis B virus, HCV: hepatitis C virus, AFP: $\alpha$-fetoprotein, NA: not available, HCC: hepatocellular carcinoma, WHO: World Health Organization. *Indicates statistical significance.

and KIF 15 expression in HCC tissues was examined using the 59 patients group. As shown in Table II, high KIF15 expression was positively associated with larger tumor size and more poorly histological differentiation (Chi-square test, $p<0.05, p<0.05$, respectively).
KIF15 expression was positively associated with the $\mathrm{MYC/E} 2 \mathrm{~F}$ target pathway in HCC tissues. To investigate the effect of KIF15 expression on inflammatory monocytes in HCC tissues, we performed GSEA using TCGA dataset. GSEA showed a significant positive correlation between KIF 15 expression and the MYC/E2F target gene set in HCC tissues (Figure 1E).

HCC cells with a high expression of MCM2 were located close to inflammatory monocytes expressing KIF15. MCM2 expression has been found superior to $\mathrm{Ki}-67$ as a marker defining the proliferative status of tumor cells and is associated with prognosis in many cancers $(28,29)$. Immunofluorescent staining showed that MCM2 was mainly stained on HCC cells around inflammatory monocytes that expressed KIF15 (Figure 2A). The number of inflammatory monocytes that expressed KIF15 was positively correlated with the number of HCC cells that expressed MCM2 (Pearson correlation coefficient $\mathrm{R}=0.818, p<0.05$ ) (Figure 2B). Proliferating cell nuclear antigen (PCNA) is a nuclear protein that is necessary for DNA synthesis and it appears in the nucleus primarily during the $\mathrm{S}$ phase of the cell cycle (30). We observed that KIF15 mRNA expression was positively correlated with $M C M 2$ and PCNA in the selected TCGA dataset (Figure 2C). These data suggest that inflammatory monocytes that expressed KIF15 promoted HCC cell proliferation.

KIF15-expressing PBMC promoted HCC cell proliferation in vitro. Based on our clinical findings described above, we hypothesized that inflammatory monocytes that expressed KIF15 promoted progression of HCC. To examine the biological significance of KIF15-expressing inflammatory monocytes close to cancer cells, we performed in vitro proliferation assays using HepG2 and $\mathrm{HuH7}$ cells co-cultured with healthy donor PBMCs in which KIF15 was expressed (Figure 3A). In colony proliferation assays (Figure 3B) and MTT assays (Figure 3C), HepG2 and $\mathrm{HuH} 7$ cells co-cultured with $\mathrm{PBMC}$ showed a high proliferation ability compared to the controls. Furthermore, western blot analysis showed that the expression of MCM2 protein was upregulated in HepG2 cells co-cultured with PBMC (Figure 3D).

\section{Discussion}

There is increasing evidence that tumor-associated inflammatory monocytes are activated by tumor cells and can facilitate tumor growth or metastasis by causing immunosuppression, angiogenesis or the epithelial-mesenchymal transition (EMT) of tumor cells in various malignancies, including HCC (31-34). For instance, TNF and IL-1 $\beta$ derived from tumor-activated monocytes synergistically induce cancer cell autophagy and EMT of cancer cells and promote tumor 
A

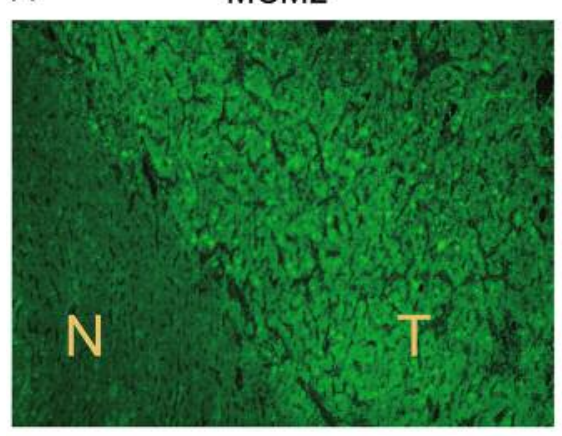

B

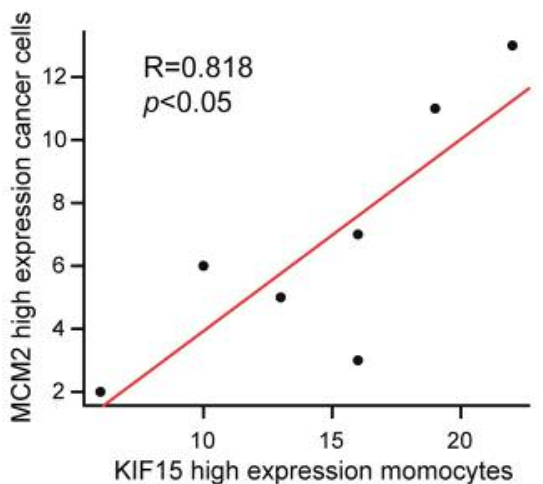

KIF15

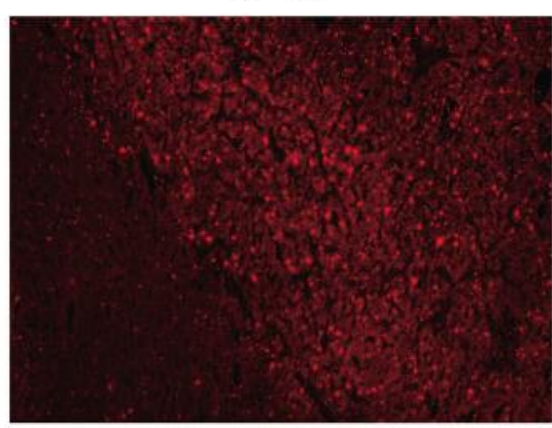

C

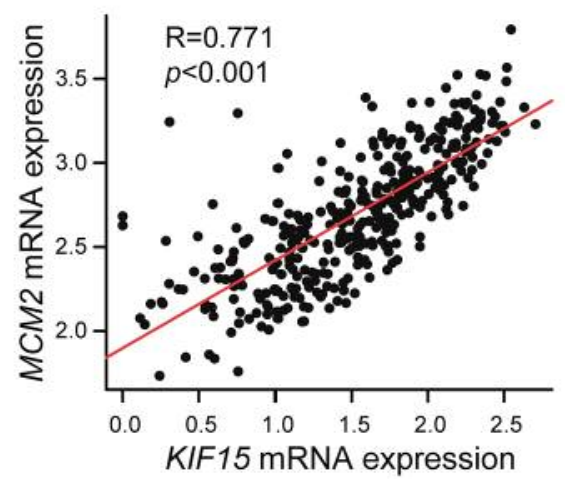

Merge
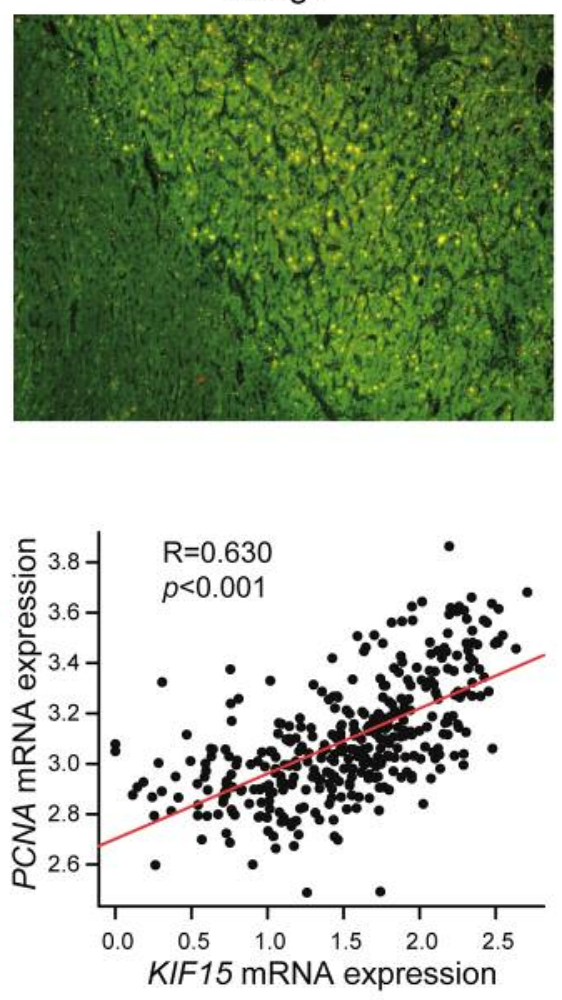

Figure 2. HCC cells with a high expression of MCM2 were located close to inflammatory monocytes that expressed KIF15. (A) Immunofluorescence images of KIF15 and MCM2 expression in HCC tissues. (B) Correlation between the number of inflammatory monocytes that expressed high levels of KIF15 and HCC cells that expressed high levels of MCM2 in HCC tissues. (C) Correlation between KIF15 mRNA expression and MCM2 or PCNA mRNA expression in the TCGA dataset.

metastasis by activating the NF-kB-SNA11 signaling pathway in the HCC microenvironment (31).

In this study, we showed that KIF15 was expressed in inflammatory monocytes around HCC cells, and its high expression predicted a poor prognosis of HCC patients. Clinicopathological analysis indicated that high expression of KIF15 in tumor tissues was positively associated with clinicopathological factors associated with tumor aggressiveness, such as tumor size and tumor differentiation in HCC. Furthermore, KIF 15 mRNA expression was significantly correlated with the $M Y C / E 2 F$ target gene set by GSEA. These results suggest that KIF15 serves as a novel prognostic biomarker in HCC patients, as well as an expression marker of tumor-associated monocytes in HCC.

Based on the clinical findings described above, we hypothesized that inflammatory monocytes that express KIF15 may promote cancer cell progression of HCC. First, in immunofluorescence staining, we observed that HCC cells with high MCM2 expression were located close to KIF15expressing inflammatory monocytes. Moreover, a positive correlation between KIF15 mRNA expression and markers of cell proliferation (MCM2 and PCNA) was observed in TCGA dataset. Next, we performed in vitro proliferation assays of HCC cells co-cultured with KIF15-expressing PBMC. As expected, proliferation ability was increased by co-culture with PBMC. These findings suggest that KIF15-expressing inflammatory monocytes could promote the proliferation of HCC cells.

There are limitations in this study. We provided clinical and biological evidence that KIF15-expressing inflammatory monocytes could promote cancer cell proliferation in HCC. Given that GSEA showed positive correlation between KIF15 mRNA expression and MYC/E2F TARGETS gene set in HCC tissues, implying that KIF15-expressing inflammatory monocytes might promote the progression of HCC via the $\mathrm{MYC} / \mathrm{E} 2 \mathrm{~F}$ pathway. However, the mechanism remains unknown. Further study will be required to elucidate the biological role of KIF15 expression in inflammatory monocytes in HCC.

In conclusion, we demonstrated that KIF15 is expressed mainly in inflammatory monocytes around HCC cells, 
A

PBMC

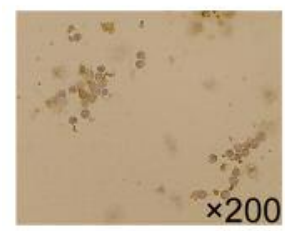

HepG2

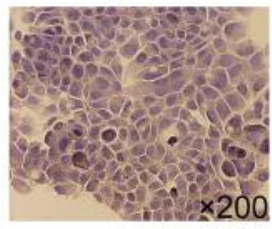

C

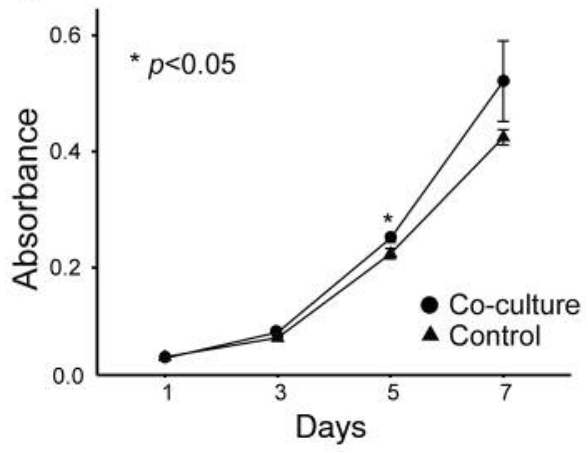

B
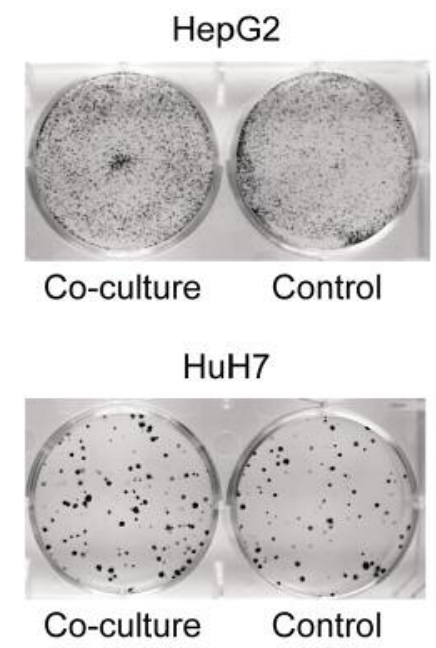

$\mathrm{HuH} 7$
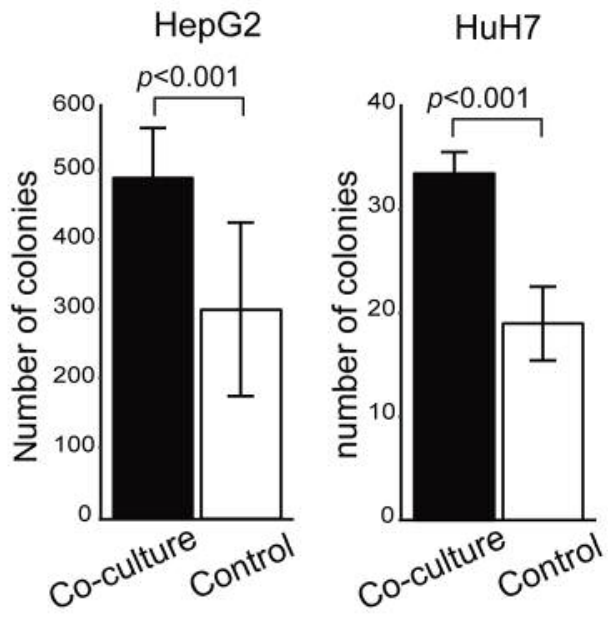

D

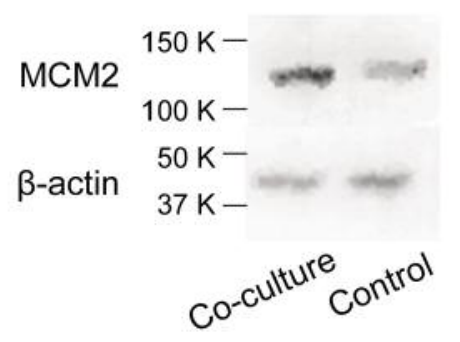

Figure 3. KIF15-expressing PBMC promoted HCC cell proliferation in vitro. Coculture of HepG2 and HuH7 cells and healthy donor PBMCs. (A) Immunohistochemical detection of KIF15 expression in PBMC and HepG2 cells. Original magnification, $\times 200$. (B) Colony proliferation assay after co-culture of HepG2 or HuH7 cells with healthy donor PBMC. (C) MTT assay after co-culture of HepG2 cells with healthy donor PBMC. (D) Western blot analysis for MCM2 expression in HepG2 cells co-cultured with healthy donor PBMC.

namely, tumor-associated monocytes. Its high expression could be a novel prognostic biomarker of poor survival of HCC patients possibly in part through promoting the proliferation of HCC cells. KIF15 might be a useful molecular therapeutic target in HCC microenvironment as its inhibition in tumor-associated monocytes could reduce cancer cell proliferation.

\section{Conflicts of Interest}

The Authors have no conflicts of interest.

\section{Authors' Contributions}

AK wrote the manuscript. AK conducted the process of experiments. AK, TM, JT, TT, MN, YK, QH, YK, YK, SK, KS, AF, YY, HW, DS, $\mathrm{YT}, \mathrm{HE}, \mathrm{YD}, \mathrm{MM}$ and $\mathrm{KM}$ designed the study and data interpretation. AK and JT prepared the organization of figures and statistical analysis.

\section{Acknowledgements}

The Authors would like to thank K. Oda, M. Kasagi, S. Sakuma, N. Mishima, T. Kawano, M. Oshiumi and M. Utou for their technical assistance. This work was supported in part by the following Grants and foundations: Japan Society for the Promotion of Science Grant-inAid for Science Research (Grant Numbers JP16K07177, JP16K10543, JP17K16454, JP17K16521, JP17K10593 and JP17K19608); OITA Cancer Research Foundation; Daiwa Securities Health Foundation; Grant-in-Aid for Scientific Research on Innovative Areas (15H0912); Priority Issue on Post-K computer (hp170227) (hp170227, hp160219); JSPS KAKENHI (15H05707); Japanese Foundation for Multidisciplinary Treatment of Cancer. HCC clinical samples were provided by the Oita Red Cross Hospital, Hiroshima Red Cross Hospital, Atomic-bomb Survivors Hospital, and Iizuka Hospital. 


\section{References}

1 Hashem B, Lau M, Eschbach K, Davila J and Goodwin J: Epidemiology of hepatocellular carcinoma in hispanics in the United States. Arch Intern Med 167(18): 1983-1989, 2007. PMID: 17923599. DOI: 10.1001/archinte.167.18.1983

2 Yopp AC and Singal AG: Epithelial to mesenchymal transition expression profiles as predictive biomarkers of recurrence following resection of HCC: Implications for current clinical use and future stratification for systemic therapy. Ann Surg Oncol 21(12): 3723-3724, 2014. PMID: 25074660. DOI: 10.1245/ s10434-014-3790-7

3 Maluccio M and Covey A: Recent progress in understanding, diagnosing, and treating hepatocellular carcinoma. CA Cancer J Clin 62(6): 394-399, 2012. PMID: 23070690. DOI: $10.3322 /$ caac. 21161

4 Hirokawa N, Noda Y, Tanaka Y and Niwa S: Kinesin superfamily motor proteins and intracellular transport. Nature Rev 10(10): 682-696, 2009. PMID: 19773780. DOI: 10.1038/nrm2774

5 Hirokawa N and Takemura R: Molecular motors and mechanisms of directional transport in neurons. Nat Rev Neurosci 6(3): 201214, 2005. PMID: 15711600 . DOI: $10.1038 / \mathrm{nrn} 1624$

6 Vale RD: The molecular motor toolbox for intracellular transport. Cell 112(4): 467-480, 2003. PMID: 12600311. DOI: 10.1016/s0092-8674(03)00111-9

7 Hirokawa N: Kinesin and dynein superfamily proteins and the mechanism of organelle transport. Science 279(23): 519-526, 1998. PMID: 9438838. DOI: 10.1126/science.279.5350.519

8 Brendza RP, Serbus LR, Duffy JB and Saxton WM: A function for kinesin $\mathrm{I}$ in the posterior transport of oskar mRNA and staufen protein. Science 289(5487): 2120-2122, 2017. PMID: 11000113. DOI: $10.1126 /$ science.289.5487.2120

9 Wang J, Guo X, Xie C and Jiang J: KIF15 promotes pancreatic cancer proliferation via the MEK-ERK signalling pathway. Br J Cancer 117(2): 245-255, 2017. PMID: 28595260. DOI: $10.1038 /$ bjc. 2017.165

10 Nagahara M, Nishida N, Iwatsuki M, Ishimaru S, Mimori K, Tanaka F, Nakagawa T, Sato T, Sugihara K, Hoon DS and Mori M: Kinesin 18A expression: Clinical relevance to colorectal cancer progression. Int J Cancer 129(11): 2543-2552, 2011. PMID: 21213216. DOI: 10.1002/ijc.25916

11 Minakawa Y, Kasamatsu A, Koike H, Higo M, Nakashima D, Kouzu Y, Sakamoto Y, Ogawara K, Shiiba M, Tanzawa H and Uzawa K: Kinesin family member 4A. A potential predictor for progression of human oral cancer. PLoS One 8(12): 1-8, 2013. PMID: 24386490. DOI: 10.1371/journal.pone.0085951

12 Yokota K, Sasaki H, Okuda K, Shimizu S, Shitara M, Hikosaka Y, Moriyama S, Yano M and Fujii Y: KIF5B/RET fusion gene in surgically-treated adenocarcinoma of the lung. Oncol Rep 28(4): 1187-1192, 2012. PMID: 22797671. DOI: 10.3892/or.2012.1908

13 Drechsler H, McHugh T, Singleton MR, Carter NJ and McAinsh AD: The Kinesin-12 Kif15 is a processive track-switching tetramer. Elife 3: 1-17, 2014. PMID: 24668168. DOI: 10.7554/eLife.01724

14 Tanenbaum ME, Macůrek L, Janssen A, Geers EF, AlvarezFernández M and Medema RH: Kif15 cooperates with Eg5 to promote bipolar spindle assembly. Curr Biol 19(20): 1703-1711, 2009. PMID: 19818618. DOI: 10.1016/j.cub.2009.08.027

15 Eskova A, Knapp B, Matelska D, Reusing S, Arjonen A, Lisauskas T, Pepperkok R, Russell R, Eils R, Ivaska J, Kaderali
L, Erfle H and Starkuviene V: An RNAi screen identifies KIF15 as a novel regulator of the endocytic trafficking of integrin. J Cell Sci 127(11): 2433-2447, 2014. PMID: 24659801. DOI: $10.1242 /$ jcs. 137281

16 Zhao H, Bo Q, Wu Z, Liu Q, Li Y, Zhang N, Guo H and Shi B: KIF15 promotes bladder cancer proliferation via the MEK-ERK signaling pathway. Cancer Manag Res 11: 1857-1868, 2019. PMID: 30881113. DOI: 10.2147/CMAR.S191681

17 Qiao Y, Chen J, Ma C, Liu Y, Li P, Wang Y, Hou L and Liu Z: Increased KIF15 expression predicts a poor prognosis in patients with lung adenocarcinoma. Cell Physiol Biochem 130021(71): 110, 2018. PMID: 30439711. DOI: 10.1159/000495155

18 Garmire LX and Subramaniam S: Evaluation of normalization methods in mammalian microRNA-Seq data. Rna 18(6): 12791288, 2012. PMID: 22532701. DOI: 10.1261/rna.030916.111

19 Komatsu H, Masuda T, Iguchi T, Nambara S, Sato K, Hu Q, Hirata H, Ito S, Eguchi H, Sugimachi K, Eguchi H, Doki Y, Mori $\mathrm{M}$ and Mimori K: Clinical significance of FANCD2 gene expression and its association with tumor progression in hepatocellular carcinoma. Anticancer Res 37(3): 1083-1090, 2017. PMID: 28314268. DOI: 10.21873/anticanres.11420

20 Komatsu H, Iguchi T, Masuda T, Hirata H, Ueda M, Kidogami S, Ogawa Y, Sato K, Hu Q, Nambara S, Saito T, Sakimura S, Uchi R, Ito S, Eguchi H, Sugimachi K, Eguchi H, Doki Y, Mori $\mathrm{M}$ and Mimori K: Attenuated RND1 expression confers malignant phenotype and predicts poor prognosis in hepatocellular carcinoma. Ann Surg Oncol 24(3): 850-859, 2017. PMID: 27770342. DOI: 10.1245/s10434-016-5573-9

21 Wakiyama H, Masuda T, Motomura Y, Hu Q, Tobo T, Eguchi H, Sakamoto K, Hirakawa M, Honda H and Mimori K: Cytolytic activity (CYT) score is a prognostic biomarker reflecting host immune status in hepatocellular carcinoma (HCC). Anticancer Res 38(12): 6631-6638, 2018. PMID: 30504371. DOI: 10.21873/anticanres. 13030

22 Kurashige J, Hasegawa T, Niida A, Sugimachi K, Deng N, Mima K, Uchi R, Sawada G, Takahashi Y, Eguchi H, Inomata M, Kitano S, Fukagawa T, Sasako M, Sasaki H, Sasaki S, Mori M, Yanagihara K, Baba H, Miyano S, Tan P and Mimori K: Integrated molecular profiling of human gastric cancer identifies DDR2 as a potential regulator of peritoneal dissemination. Sci Rep 6: 22371, 2016. PMID: 26934957. DOI: 10.1038/srep22371

$23 \mathrm{Hu}$ Q, Masuda T, Sato K, Tobo T, Nambara S, Kidogami S, Hayashi N, Kuroda Y, Ito S, Eguchi H, Saeki H, Oki E, Maehara $\mathrm{Y}$ and Mimori K: Identification of ARL4C as a peritoneal dissemination-associated gene and its clinical significance in gastric cancer. Ann Surg Oncol 25(3): 745-753, 2018. PMID: 29270876. DOI: 10.1245/s10434-017-6292-6

24 Masuda T, Shinden Y, Noda M, Ueo H, Hu Q, Yoshikawa Y, Tsuruda Y, Kuroda Y, Ito S, Eguchi H, Ohno S and Mimori K: Circulating pre-microRNA-488 in peripheral blood is a potential biomarker for predicting recurrence in breast cancer. Anticancer Res 38(8): 4515-4523, 2018. PMID: 30061217. DOI: 10.21873/anticanres.12755

25. Shimizu D, Masuda T, Sato K, Tsuruda Y, Otsu H, Kuroda Y, Eguchi H, Kodera Y and Mimori K: CRMP5-associated GTPase (CRAG) is a candidate driver gene for colorectal cancer carcinogenesis. Anticancer Res 39(1): 99-106, 2019. PMID: 30591445. DOI: 10.21873/anticanres.13084

26 Nambara S, Masuda T, Nishio M, Kuramitsu S, Tobo T, Ogawa Y, Hu Q, Iguchi T, Kuroda Y, Ito S, Eguchi H, Sugimachi K, 
Saeki H, Oki E, Maehara Y, Suzuki A and Mimori K: Antitumor effects of the antiparasitic agent ivermectin via inhibition of Yesassociated protein 1 expression in gastric cancer. Oncotarget 8(64): 107666-107677, 2017. PMID: 29296196. DOI: 10.18632/oncotarget.22587

27 Mizuno H, Kitada K, Nakai K and Sarai A: PrognoScan : a new database for meta-analysis of the prognostic value of genes. BMJ Med Genomics 2: 18, 2009. PMID: 19393097. DOI: $10.1186 / 1755-8794-2-18$

28 Huang H, Huang W, Lin C, Eng HL, Li SH, Li CF, Lu D, Yu SC and Hsiung CY: Immunohistochemical expression of p16 INK4A, Ki-67, and $\mathrm{Mcm} 2$ proteins in gastrointestinal stromal tumors: Prognostic implications and correlations with risk stratification of NIH consensus criteria. Ann Surg Oncol 13(12): 1633-1644, 2006. PMID: 17013685. DOI: 10.1245/s10434-0069188-4

29 Yousef EM, Furrer D, Laperriere DL, Tahir MR, Mader S, Diorio $\mathrm{C}$ and Gaboury LA: MCM2: An alternative to Ki-67 for measuring breast cancer cell proliferation. Mod Pathol 30(5): 682-697, 2017. PMID: 28084344. DOI: 10.1038/modpathol.2016.231

30 Mathews MB, Bernstein RM, Franza BR Jr and Garrels JI Identity of the proliferating cell nuclear antigen and cyclin: Nature 24(306): 374-376, 1984. PMID: 6145097. DOI: $10.1038 / 309374 \mathrm{a} 0$

31 Chen DP, Ning WR, Li XF, Wei Y, Lao XM, Wang JC, Wu Y and Zheng L: Peritumoral monocytes induce cancer cell autophagy to facilitate the progression of human hepatocellular carcinoma. Autophagy 14(8): 1335-1346, 2018. PMID: 29940792. DOI: $10.1080 / 15548627.2018 .1474994$
32 Kapanadze T, Gamrekelashvili J, Ma C, Chan C, Zhao F, Hewitt S, Zender L, Kapoor V, Felsher DW, Manns MP, Korangy F and Greten TF: Regulation of accumulation and function of myeloid derived suppressor cells in different murine models of hepatocellular carcinoma. J Hepatol 59(5): 1007-1013, 2013. PMID: 23796475. DOI: 10.1016/j.jhep.2013.06.010

33 Wan S, Zhao E, Kryczek I, Vatan L, Sadovskaya A, Ludema G, Simeone DM, Zou W and Welling TH: Tumor-associated macrophages produce interleukin 6 and signal via STAT3 to promote expansion of human hepatocellular carcinoma stem cells. Gastroenterology 147(6): 1393-1404, 2014. PMID: 25181692. DOI: $10.1053 /$ j.gastro.2014.08.039

34 Menen RS, Hassanein MK, Momiyama M, Suetsugu A, Moossa AR, Hoffman RM and Bouvet M: Tumor-educated macrophages promote tumor growth and peritoneal metastasis in an orthotopic nude mouse model of human pancreatic cancer. In Vivo 26(4): 565-569, 2012. PMID: 22773569.
Received November 12, 2019

Revised December 1, 2019

Accepted December 4, 2019 